\title{
Detection of extraneous signals during sentence recognition 1
}

\author{
V. M. HOLMES, ${ }^{2}$ UNIVERSITY OF MELBOURNE \\ AND K. I. FORSTER, MONASH UNIVERSITY
}

\begin{abstract}
In two separate experiments, $40 \mathrm{Ss}$ were presented with recorded sentences during each of which a click occurred. Ss had to depress a key as soon as they heard the click. RTs were faster when the click was located at the major syntactic break of the sentence compared with RTs to clicks not at a break. This confirmed the hypothesis that processing load is a function of the surface structure of sentences, although the role of minor breaks was not clear. A second finding was that RTs were slower when the click was in the first rather than in the second half of the sentence. This can also be explained in terms of differential processing loads.
\end{abstract}

The results of a recent experiment by Fodor and Bever (1965) have suggested that the perception of an extraneous signal during sentence recognition is influenced by the location of the signal in relation to the surface structure of the sentence. Ss were required to listen to a series of recorded sentences, on each of which an extraneous signal (a click) was superimposed. After each presentation, Ss had to write the sentence out indicating where they thought the click occurred. The results showed that there was a high proportion of erroneous responses. When these errors were examined, there was a significant tendency for them to be displaced towards the major syntactic break.

Additional support for this finding was provided by Garrett, Bever, and Fodor (1966), using the same methodology but a different method of analyzing the data. Their experiment was designed to rule out an interpretation of the "click migration" effect as being due to the influence of intonational cues in the sentences and not the constituent structure.

In both these studies, the results were interpreted as confirmation of the hypothesis that the immediate constituent functions in some sense as the perceptual unit in sentence decoding. The conclusion was based on the assumption of "the tendency for a perceptual unit to preserve its integrity by resisting interruptions (Fodor \& Bever, 1965, p. 415)." Judging from the experimental procedure adopted, Fodor and Bever evidently intended the term "immediate constituent" to refer to such relatively higher-order constituents as clauses or verb phrases.
Fodor and Bever (1965) and Garrett et al (1966) assumed that S's judgments of the click's position were an accurate reflection of S's perception of the location of the click in relation to the sentence. But it can be argued that having to recall the sentence and write it out could have interfered with $S$ 's retention of the location of the click. Thus, the errors might not have indicated factors operating while the sentence was being perceived, but might have been due to events occurring subsequent to the perceptual analysis.

The possible role of memory variables in click location was later recognized by Fodor, Bever, and Garrett. ${ }^{3}$ They conducted an experiment in which Ss were presented with the same sentence twice in succession, each version of which had a click superimposed on it. Ss were required to state: (1) whether the two sentences were the same or different, and (2) whether the clicks' positions were the same or different. More "same" click location judgments were found where a major constituent boundary intervened between the two different click positions.

Fodor et al argued that this method showed that errors of click location do occur during sentence processing, since it "requires Ss to operate with only aural input, requires no delay in recording responses, and eliminates any requirement that the $S$ reproduce the sentences being tested (p. 68). ${ }^{3}$ However, the task introduces additional sources of interference to accurate reporting of the subjective click location. Not only had the $S$ to listen to and recall two sentences, but he had to decide whether they were the same or different, in addition to estimating and recalling two click locations and deciding whether they were the same or different. It seems that it is inherently impossible to separate out the effects of memory and a true perceptual effect using the click location method.

Underlying the theoretical account of the click-location results is the assumption that the processing load during a constituent must be greater than at the end of it. Consistent with this assumption is a subsidiary finding, reported by Fodor and Bever (1965), that clicks that occurred at major constituent boundaries were more accurately located than clicks that were not at constituent boundaries. In order to test more directly the hypothesis that processing load decreases at major constituent boundaries, it was decided simply to require $S$ s to detect the click and to indicate this as quickly as possible by a simple motor response. If the click-location result were due only to memory variables, then no differences in reaction times (RTs) to the clicks would be expected. However, if perceptual effects during processing really do occur, then RTs could hardly fail to be affected by them.

\section{EXPERIMENT 1}

On the assumption that the processing load during a major constituent is heavier than at the end of a major constituent, it follows that detection time (and hence RT) for clicks not at breaks should be slower than detection time for clicks at major breaks. The aim of the present experiment was to test this hypothesis. Sentences were constructed where the click interrupted either a large number of constituents or no constituents, e.g., between clauses. Indicating the location of the click by a slash, an example of the former condition is: After the coroner's inquest is held, there will be a complete/ inquiry into yesterday's tragedy; an example of the latter: The little boys were very tired/, so they were unable to move the heavy boulder. A third condition was included, where the click interrupted an intermediate number of constituents, occurring at what appeared to be a "minor break" in the sentence structure, e.g., After all the students had deserted the university buildings, many academics/ assembled for the conference. It was thought that RTs to clicks at a minor break might also be faster than RTs to clicks not at a break.

\section{Subjects}

The Ss were 40 male and female undergraduates from the University of Melbourne, who were paid for their participation in the experiment. All were native speakers of English.

\section{Apparatus}

A four-track stereophonic tape recorder was used to record on one track 24 sentences of constant 15-word length. The sentences were spoken with normal intonation except that an effort was made to avoid pauses between words. On the second track, a short burst of noise, a "click," about $80 \mathrm{msec}$ in duration, was 
recorded for each sentence. When the tape was played, the onset of the click set off a voice-key that activated a chronoscope, which in turn was stopped by the depression of a key by the $S$. These RTs were measured to the nearest 100 th of a second.

\section{Design}

All 24 sentences had a major syntactic break separating two clauses, i.e., S-dominated constituents. The main treatment was the position of the click in relation to the constituent structure. The click was located at a major break, at a minor break, or not at a break. The average separations of the click and the major break for the latter two conditions were 6.3 and 16.9 syllables respectively, where the boundaries between adjacent words are counted as syllables. Thus, the conditions were not directly comparable with those in the click-location studies, in which the clicks were generally located within four or five syllables of the major break. A second factor was whether the click occurred during the first or the second half of the sentence.

Each of 16 of the sentences was constructed so that it possessed both a major and a minor break in either its first or second half. Two cross-recordings of these 16 sentences were prepared. One set of the sentences had the click at the minor break, and in the other set, the click was at the major break. This was to compare the effect of having the click at a minor or major break for the same sentence and within the one-sentence position. Since Ss underwent all conditions, two groups of Ss were needed to prevent practice effects from presentation of the same sentence twice to an $S$. The remaining eight sentences had clicks not at a break and were the same for both groups. Thus, for each group of 20 Ss there were four sentences in each of 3 by 2 conditions. The sentences, with click positions indicated, are in the Appendix.

The two lists of 24 sentences were randomized and divided into four blocks of six. The four blocks were randomized and rearranged according to a Latin square design. This gave four orders that were given to four consecutive Ss and then repeated five times for each group of Ss. Half the Ss in each group were presented with sentences to the right ear and clicks to the left ear, and vice versa for the other half.

An additional control was employed to eliminate the possibility that faster RTs might occur to clicks that were at acoustic pauses in the sentences. The energy output of the two tracks was analyzed using a two-channel pen-recorder, so that the speech energy level coincident with the click could be determined. The sentences were re-recorded until only 4 of the 48 sentences had clicks occurring at drops in the energy level. One sentence had the click not at a break, in one the click was at a minor break, and the other two had the click at a major break. It was felt that the influence of these four sentences would be minimal over the three conditions. All the clicks were at the end of a word.

\section{Procedure}

The Ss, who were tested individually, were presented with the material dichotically through headphones. They were given practice in responding to clicks in isolation. There were four practice items, followed by the 24 experimental sentences for each group. Ss listened to each sentence, depressed the key as quickly as they could on hearing the click, and then wrote the sentence down as accurately as they could. Writing the sentence out was to insure that Ss were actively decoding the sentences and not simply paying attention to the click.

\section{Results and Discussion}

For each S, the mean of the RTs for the four sentences in each condition was calculated. The pattern of results for each group was reasonably similar, though overall RT was slightly longer for Group 2. The mean RTs over both groups of Ss are presented graphically in Fig. 1. RTs to clicks in the first half of the sentence seemed to be slower than RTs to clicks in the second half of the sentence. The hypothesis that RTs to clicks at a major

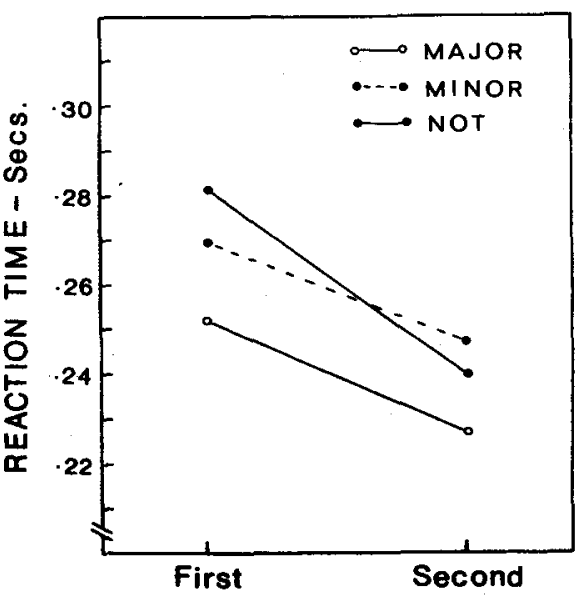

HALF OF SENTENCE

Fig. 1. Mean RT in Experiment 1 as a function of position of the signal in relation to the sentence, and relative to the constituent structure (at a major break, at a minor break, and not at a break). break would be faster than RTs to clicks not at a break seemed to be confirmed.

A four-way analysis of variance was performed on the data, the conditions being sentence position, constituent position, Ss, and groups (Winer, 1962, pp. 319-325). Setting $\alpha=.05$, the main effect of constituent position was significant, $[\mathrm{F}(2,76)=5.52]$, as was sentence position, $[F(1,38)=26.67]$, while groups was not significant $[F(1,38)=4.05]$. None of the interaction terms was significant: for Constituent Position by Groups, $F(2,76)=1.32$; for Sentence Position by Groups, $F(1,38)=0.11$; for Constituent Position by Sentence Position, $F(2,76)=1.53$; and for Constituent Position by Sentence Position by Groups, $\mathrm{F}(2,76)=1.11$.

Although the main effect of constituent position was significant, the two specific predicted comparisons need to be tested (Winer, 1962, pp. 207-211). While the mean RT to clicks at a major break was significantly faster than the mean RT to clicks not at a break, $[F(1,76)=14.12]$, the mean RT to clicks at a minor break was not significantly different from the mean RT to clicks not at a break $[F(1,76)=0.18]$. This would seem to suggest that the constituent must be defined at the deepest level of the structure for an effect to be obtained.

It could be argued that the effects observed may have been minimized by a tendency for Ss to abandon temporarily the sentence-processing task in order to handle the click more efficiently. This would lead one to expect a positive correlation between RT and accuracy of recall of the sentence, since interruption of decoding would presumably produce errors in perception.

The reproduced sentences were scored for accuracy of recall in terms of number of correct words. The relevant data were the individual sentences, as correlations cannot be based on more than one score from each $\mathbf{S}$ for a given variable. Thus, for each sentence, the 20 RTs and corresponding accuracy of recall scores were correlated. Using the $Z$ transformation, the average correlation was -.061 for Group 1 and -.012 for Group 2 , neither of which was significant at the .05 level, with $\mathrm{df}=18$. Of the 48 individual correlations, 2 were significant, but at least 1 in 20 correlations would be expected to be significant, in any case. Thus, it seemed clear that RT and accuracy of recall were not correlated in this task. The fact that recall of the sentences was very good (a total mean of 14.2 words out of 15), indicated that Ss must have been decoding the auditory stimuli as sentences rather than as unstructured sequences. 


\section{EXPERIMENT 2}

Although the findings of Experiment 1 seem relatively clear-cut, there was a reason for treating the results with some scepticism. In preliminary work, conflicting results had been obtained. In one case, the major results of Experiment 1 were confirmed, and in the other, the findings were inconclusive. ${ }^{4}$ In addition, the fact that RTs to clicks at minor breaks were not significantly faster than RTs to clicks not at breaks was surprising. Thus, the same hypotheses as in Experiment 1 were tested with a different sample of Ss.

\section{Subjects}

There were $40 \mathrm{Ss}$, all native speakers of English, who were graduate and undergraduate psychology students. Sixteen Ss were volunteers; 24 Ss were paid for their participation.

\section{Apparatus, Design, and Procedure}

Since the stimulus material was the same as in Experiment 1, the apparatus, design, and procedure were also exactly the same as for Experiment 1.

\section{Results and Discussion}

Inspection of Fig. 2 indicates three main points. (1) In agreement with the results of Experiment 1, overall RT to clicks at a major break was faster than RT to clicks not at a break. (2) However, it is obvious that this effect has not occurred when the click was located in the second half of the sentence. (3) For the first half of the sentence, RTs to clicks at a minor break were intermediate between the other two

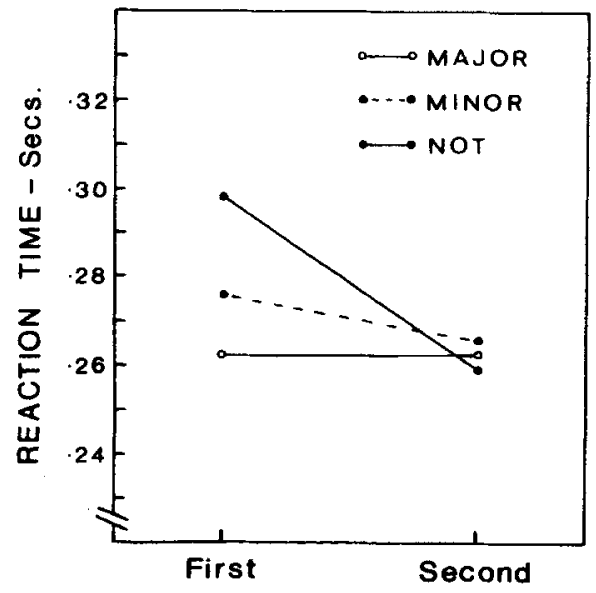

HALF OF SENTENCE

Fig. 2. Mean RT in Experiment 2 as a function of position of the signal in relation to the sentence, and relative to the constituent structure (at a major break, at a minor break, and not at a break). conditions. A similar trend can be observed in the results of Experiment 1.

As would be expected from the marked reduction of any effects in the second half of the sentence, the results of the four-way analysis of variance revealed a significant interaction between Constituent Position and Sentence Position $[F(2,76)=7.83$, with $\alpha=.05]$. Both main effects of constituent position $[F(2,76)=5.00]$ and sentence position $[F(1,38)=9.77]$ were significant, while the main effect of groups was not significant $[F(1,38)=0.99]$. The remaining testable interaction terms were not significant: for Constituent Position by Groups, $\quad F(2,76)=1.54 ;$ for Sentence Position by Groups, $F(1,38)=1.50$; and for Constituent Position by Sentence Position by Groups, $F(2,76)=0.02$.

The predicted comparison that RTs at major breaks would be faster than RTs not at breaks was significant $[F(1,76)=7.20]$. However, it is clear that the only points contributing to the effect were those in the first half of the sentence. The second predicted comparison between RTs at minor breaks and RTs not at breaks was again not significant $[F(1,76)=1.80]$.

As in Experiment 1, accuracy of recall of the sentences was again examined. The overall mean accuracy of recall score was 14.2 , an average error of only one word. Six of the correlations of accuracy of recall with RT for the sentences were significant $(\mathrm{df}=18, \alpha=.05)$, three in Group 1 and three in Group 2. These correlations were all negative. Moreover, the average correlations, using the $\mathrm{Z}$ transformation, were -.141 and -.129 , neither of which was significant. It was concluded that accuracy of recall was not correlated with length of RT and was thus not reducing RT differences.

\section{GENERAL DISCUSSION}

The results of both experiments were consistent with one another in showing that clicks at major breaks are detected more rapidly than clicks not at breaks. It would be difficult to attribute this result to anything but the differential amount of processing required during a major constituent compared with processing at a constituent boundary.

The second main finding was that RTs were slower in the first, compared with the second, half of the sentence. This could be explained by using the same assumption that RT is a function of amount of interference from sentence decoding. At the beginning of the sentence, the uncertainty regarding the nature of the sentential input is maximal, while by the middle or end of the sentence the possibilities have to a large extent become predictable. Thus, the decoding load would be greater earlier in the sentence. This indicates that the effects of left-to-right constraints on sentence recognition may be as marked as the effects of hierarchical structure. Marks (1967) has reported findings that also imply this conclusion.

Alternatively, if the commencement of the sentence is considered to be a warning signal, then the slower RT in the first half of the sentence could be a result of Ss' not being prepared for the signal when it occurred soon after the start of the sentence. However, against this interpretation is the finding that simple RT does not vary as a function of foreperiod length when the foreperiod varies irregularly (Woodworth \& Schlosberg, 1955).

In Experiment 2, the difference between RTs at major breaks and RTs not at breaks in the first half of the sentence was $.036 \mathrm{sec}$, but the means were virtually the same in the second half of the sentence. This contributed to the significant interaction, a result that was not obtained in Experiment 1. However, there was a similar trend in Experiment 1; in the first half of the sentence, the difference of $.030 \mathrm{sec}$ was reduced to $.014 \mathrm{sec}$ in the second half of the sentence. Explanation of this result does not necessitate any new assumptions. If overall processing load decreases toward the end of the sentence, then a click occurring within a constituent in the second half of the sentence becomes more like a click at a break the nearer it is to the end of the sentence. Since the structural information conveyed by the last few words would tend to be highly predictable, processing load would be minimal.

A final point that warrants discussion concerns the kind of constituent that produces an effect on RTs. In both experiments, RTs at minor breaks were overall, slightly but not significantly, faster than RTs not at breaks, although in the first half of the sentence there seemed to be a substantial difference between the two conditions. 5 This trend would be consistent with the hypothesis put forward in this paper that processing load is a function of the number of constituents of the surface structure disrupted by the click's location. On the other hand, it could be that the crucial requirement is that the constituent be S-dominated, since this was true of all major breaks and for some of the minor breaks. On this view, the reduced effect for minor breaks could be due to the fact that only some of the minor breaks produced an effect, i.e., those that were S-dominated. (Nevertheless, clicks located at these breaks still satisfied the condition that several constituents be interrupted, e.g., We thought/ that he 
would die, but we had not expected such amazingly strong willpower.) If this argument were correct, then it might be expected that RTs to clicks at S-dominated minor breaks would be faster than RTs to clicks at the other minor breaks.

Unfortunately, six of the eight S-dominated minor breaks had clicks in the first half of the sentence, and six of the eight not S-dominated minor breaks had clicks in the second half of the sentence. Thus, this classification of minor breaks was confounded with sentence position. For Experiment 1, the mean RT for S-dominated minor breaks was $.260 \mathrm{sec}$, compared with $.255 \mathrm{sec}$ for other minor breaks; for Experiment 2, the means were .268 and $.275 \mathrm{sec}$, respectively. Only in Experiment 2 were the means in the right direction, and over both experiments the difference was negligible. Of course, this finding of no difference is equivocal, as it is possible that the sentence position effect may have cancelled out any superiority of the S-dominated minor breaks. Clearly, future research would be required to resolve this issue.

It may be concluded that detection time for clicks not at breaks was slower than detection time for clicks at major breaks. This effect was stronger where the clicks were located in the first, rather than in the second, half of the sentence. However, the role of minor breaks was not clearly determined.

It could be argued that this finding revealed a perceptual basis for the click-migration effect reported in the click-location studies. The fact that the perception of a click located within a constituent was delayed could be taken as an indication that the $S$ did not perceive the click until the end of the constituent had been reached, thus producing the migration effect. But this cannot be correct for the following reasons: (1) The largest difference between RTs not at a break and RTs at a major break was $.036 \mathrm{sec}$. This difference corresponds to approximately one-third of a syllable and is far too small to suggest that detection of clicks not at breaks was consistently delayed to the major constituent boundary. (2) The argument assumes that clicks not at a break were always reported as occurring subsequent to their actual location. However, the results of the click-location experiments showed that clicks were attracted to the nearest constituent boundaries, whether these were before or after the click.

The results of the present study do demonstrate that perceptual effects must underlie the differences in accuracy of click location observed by Fodor and Bever (1965). The delay in detection time for clicks not at breaks must be a reflection of increased sentence-processing load. It seems reasonable to infer that this greater processing load causes the $S$ to be unclear as to the exact location of the click relative to the sentential input, resulting in less accurate judgments.

\section{APPENDIX \\ Sentences for Experiments 1 and 2 with Click Positions Indicated by Slash Marks}

Clicks at either a major break $(/)$ or a minor break $\left(/^{*}\right)$ in the first half of the sentence:

(1) The ghastly war/* was over/, and they were now free to return to their homeland.

(2) According to the papers/* it was suicide/, but we believe the evidence strongly indicates murder.

(3) The little boys/* were very tired/, so they were unable to move the heavy boulder.

(4) We thought/* that he would die/, but we had not expected such amazingly strong willpower.

(5) We admired him/, but we felt/* that there were often complex motivations underlying his behavior.

(6) Since Caesar was dead/, it seemed/* that Antony would assume supreme power of the empire.

(7) Because it was raining/, they decided $/{ }^{*}$ to hold the meeting indoors in the large hall.

(8) When they arrived/, they found/* the woman was screaming and in a state of hysteria.

Clicks at either a major break (/) or a minor break $\left(/^{*}\right)$ in the second half of the sentence:

(9) After all the students had deserted the university buildings/, many academics/ ${ }^{*}$ assembled for the conference.

(10) Although they have gained some degree of sexual liberty/, women/* still remain restricted by conventions.

(11) The full effects of industrialization were not yet realized/, but by $1900 / *$ they were perceptible.

(12) Since the political attitudes of Australians are confused and ambivalent/, the study/* was made difficult.

(13) He waited confidently for the appreciative burst of laughter/* that would follow/, but none came.

(14) Radical improvements should be made to the system/* within a few months/, the minister said.

(15) Although their hopes of finding the three missing men/* were dwindling/, the search was resumed.

(16) The divergence of views in the party did not diminish $/^{*}$ its influence/, as he claimed.

Clicks not at a break in the first half of the sentence:

(17) Just as the/ solicitor had exhausted his stock of convincing arguments, they decided to agree.

(18) Nothing unexpected happened in the/ rest of the cross-examination, but it still ended very late.

(19) When he discovered that there/ was inadequate provision for study or research, he promptly resigned.

(20) If they are/ kept continually on the required deprivation schedule, the animals become very excitable.

Clicks not at a break in the second half of the sentence:

(21) Though some modern anthropologists may not appreciate the novel, the author has/ done conscientious research.

(22) After the coroner's inquest is held, there will be a complete/ inquiry into yesterday's tragedy.

(23) We left the room, for we felt disgusted by the excessive/ sentimentality of the participants.

(24) As they crouched there, the dark swamp resounded to the shouting/ voices and barking hounds.

\section{REFERENCES}

FODOR, J. A., \& BEVER, T. G. The psychological reality of linguistic segments. Journal of Verbal Learning \& Verbal Behavior, $1965,4,414-420$.

GARRETT, M., BEVER, T. G., \& FODOR, J. A. The active use of grammar in speech perception. Perception \& Psychophysics, 1966, 1, 30-32.

MARKS, L. E. Some structural and sequential factors in the processing of sentences. Journal of Verbal Learning \& Verbal Behavior, 1967, 6, 707-713.

WINER, B. J. Statistical principles in experimental design. New York: McGraw-Hill, 1962.

WOODWORTH, R. S., \& SCHLOSBERG H. Experimental psychology. (3rd ed.) New York: Holt, 1955.

\section{NOTES}

1. This research was carried out by the first author, as a PhD student at the University of Melbourne, under the supervision of the second author.

2. Address: University of Melbourne, Parkville, Victoria, Australia, 3052.

3. Fodor, J. A., Bever, T. G., \& Garrett, M. The development of psychological models for speech recognition. M.I.T. Tech. Report No. 19(628)-5705, 1968.

4. It is worthwhile reporting that, in the pilot study, RTs to clicks in isolation with no warning signals were significantly faster than RTs to clicks during sentences. This finding guaranteed that sentence processing did actually lengthen detection time.

5. It is of interest to note that in three cases out of the eight sentences with the click at a minor break, the $S$ could have responded as though the click were at a major break. This 
poscibility arises because it was not until the remsinder of the sentence had been presented that the nature of the breat could be determined. The three sentences (i.e., 1, 2, and 3 in the Appendix) might have been expected to have shorter RTs then the other sentences with the click at a mino batk since these senteaces were all in the first haif of the sentence, the proper comparison was within this condition. Bower. this effoct did not occur. The mean RT for
Sentences 1, 2, and 3 was .270 sec for Experiment 1 and $280 \mathrm{sec}$ for Experiment 2, compered with mean RTs of .269 and .274 sec for the other minor-breal sentences.

(Accopted for pubticotion Augurt 15. 1969.1 